\title{
Interhemispheric connectivity influences the degree of modulation ofTMS-induced effects during auditory processing
}

\author{
Jamila Andoh ${ }^{1,2 *}$ and Robert J. Zatorre ${ }^{1,2 *}$ \\ Montreal Neurological Institute, Mc Gill University, Montreal, QC, Canada \\ 2 International Laboratory for Brain, Music, and Sound, Montreal, QC, Canada
}

Edited by:

Mari Tervaniemi, University of Helsinki,

Finland

\section{Reviewed by:}

Kimmo Alho, University of Helsinki,

Finland

Séverine Samson, Université de Lille,

France

Tom Ethofer, University Tubingen,

Germany

\section{*Correspondence:}

Jamila Andoh and Robert J. Zatorre,

Montreal Neurological Institute, 3801

University Street, Montreal, QC,

Canada H3A $2 B 4$.

e-mail: jamila.andoh@mcgill.ca;

www.zlab.mcgill.ca
Repetitive transcranial magnetic stimulation (rTMS) has been shown to interfere with many components of language processing, including semantic, syntactic, and phonologic. However, not much is known about its effects on nonlinguistic auditory processing, especially its action on Heschl's gyrus (HG). We aimed to investigate the behavioral and neural basis of rTMS during a melody processing task, while targeting the left $\mathrm{HG}$, the right $\mathrm{HG}$, and the Vertex as a control site. Response times (RT) were normalized relative to the baseline-rTMS (Vertex) and expressed as percentage change from baseline (\%RT change). We also looked at sex differences in rTMS-induced response as well as in functional connectivity during melody processing using rTMS and functional magnetic resonance imaging (fMRI). $f M R I$ results showed an increase in the right $\mathrm{HG}$ compared with the left $\mathrm{HG}$ during the melody task, as well as sex differences in functional connectivity indicating a greater interhemispheric connectivity between left and right HG in females compared with males. TMS results showed that $10 \mathrm{~Hz}-\mathrm{rTMS}$ targeting the right $\mathrm{HG}$ induced differential effects according to sex, with a facilitation of performance in females and an impairment of performance in males. We also found a differential correlation between the \%RT change after $10 \mathrm{~Hz}-\mathrm{rTMS}$ targeting the right $\mathrm{HG}$ and the interhemispheric functional connectivity between right and left $\mathrm{HG}$, indicating that an increase in interhemispheric functional connectivity was associated with a facilitation of performance. This is the first study to report a differential rTMS-induced interference with melody processing depending on sex. In addition, we showed a relationship between the interference induced by rTMS on behavioral performance and the neural activity in the network connecting left and right $\mathrm{HG}$, suggesting that the interhemispheric functional connectivity could determine the degree of modulation of behavioral performance.

Keywords: repetitive transcranial magnetic stimulation, functional magnetic resonance imaging, interhemispheric functional connectivity, Heschl's gyrus, melody processing

\section{INTRODUCTION}

Specialization and interhemispheric asymmetries of auditory cortical function have been examined using a variety of techniques. It has long been thought that the right auditory cortex is especially important for the processing of melodic information (Zatorre et al., 2002) whereas the left is more critical for speech processing (Hickok and Poeppel, 2007). This asymmetry can be explained on the basis of hemispheric differences in spectral and temporal resolution (Tallal et al., 1993; Zatorre et al., 2002; Poeppel, 2003) and is supported by considerable empirical data from neuroimaging studies (Boemio et al., 2005; Schonwiesner et al., 2005; Jamison et al., 2006; Giraud et al., 2007; Hyde et al., 2008). However, imaging techniques lack the ability to demonstrate causal inference, and therefore lesion evidence becomes important. Although many lesion studies do show that right auditory cortex is critical for certain aspects of pitch discrimination (Zatorre, 1988; Peretz, 1990; Stewart et al., 2006), the anatomical precision of such studies is often limited. In particular, lesion studies alone cannot probe the way in which the lesion may have caused reorganization or changes in interhemispheric functional processing.
Repetitive transcranial magnetic stimulation (rTMS) has been proposed as a "virtual lesion" approach, and has the potential to produce both anatomical precision and, when coupled with functional neuroimaging, to allow the investigation of changes in functional organization. Several prior studies have used rTMS to investigate the processing of auditory language. Knecht et al. (2002) applied rTMS over the left temporoparietal area and found a disruption of behavioral performance [i.e., increase of response time (RT)] during a picture-word verification task. These authors also reported a correlation between verbal disruption and the degree and side of lateralization as measured using functional transcranial Doppler sonography during a word-generation task: participants with greater bilaterality were less affected by either left- or right-sided TMS than participants with strong lateralization to one hemisphere. Other studies reported a facilitation effect of rTMS applied over the left temporoparietal area, i.e., decreased RT during an auditory task of word recognition (Andoh et al., 2006, 2008; Andoh and Paus, 2011). Whether the effects of rTMS on behavior are disruption or facilitation is thought to depend on a number of factors whose complex interplay is not well understood; stimulation frequency appears to be one of the most important factors in this regard (Pascual-Leone et al., 1998; Hallett, 2000; Andoh et al., 
2008). Indeed, rTMS frequency has been shown to be bidirectional in motor cortex studies, with low-frequency rTMS $(<1 \mathrm{~Hz})$ inducing long-lasting depression of motor-evoked potentials (Wassermann et al., 1996; Chen et al., 1997) and high-frequency rTMS (>5 Hz) inducing an increase of corticospinal excitability (Berardelli et al., 1998; Peinemann et al., 2004); however its effect on non-motor areas are less clear (Andoh et al., 2008).

Here, we aimed to investigate the behavioral and neural basis of rTMS-mediated auditory phenomena in the context of melody processing in order to test the hypothesis that the right auditory cortex is more critical than the left for this function. In order to localize the relevant auditory activity to use as a target for TMS in each participant, we used a melody discrimination task (Foster and Zatorre, 2010) that has shown a significant bilateral hemodynamic response in temporal auditory areas, including Heschl's gyrus (HG). We were also interested in investigating the effect of rTMS frequency ( 1 and $10 \mathrm{~Hz}$ ). Based on previous work in melody processing showing the crucial role of the right auditory cortex (Zatorre et al., 2002), and based on previous rTMS results on language processing, we expected that 1 and $10 \mathrm{~Hz}-\mathrm{rTMS}$ targeting the right $\mathrm{HG}$ would facilitate performance during a melody discrimination task compared with rTMS targeting the left HG. We also expected $1 \mathrm{~Hz}-\mathrm{rTMS}$ to facilitate more than $10 \mathrm{~Hz}-\mathrm{rTMS}$ by analogy with previous results (Andoh et al., 2006; Andoh and Paus, 2011).

In addition, based on previous literature showing sex differences in anatomy and function within HG and planum temporale (Kulynych et al., 1994; Good et al., 2001), as well as sex differences in interhemisheric functional connectivity in the superior temporal cortex (Kulynych et al., 1994; Good et al., 2001; Bitan et al., 2010), we examined sex differences in functional magnetic resonance imaging (fMRI) results as well as in TMS-induced modulation on behavioral performance. Furthermore, we investigated if there was a relationship between the pattern of rTMS results and the fMRI-based functional connectivity between right and left auditory cortices. This question was predicated on previous studies supporting the hypothesis that some brain functions may operate in a state of interhemispheric compensation. That is, the result of rTMS-induced modulation may reflect not only the local effect on the area targeted, but also adaptive plasticity in the opposite hemisphere for functional recovery (Thiel et al., 2006a; O'shea et al., 2007). Therefore, we sought to determine if the behavioral effect of rTMS was influenced by the degree of functional connectivity between auditory regions.

\section{MATERIALS AND METHODS PARTICIPANTS}

Sixteen healthy volunteers (eight male) with normal hearing were recruited (mean age $\pm S D, 21.6 \pm 2.0$ years old). Participants spent (mean score $\pm S D$ ) $2.7 \pm 1.1$ years in university and had minimal formal musical training as measured by a self-reported history of musical experience, indicating less than 1 year of musical experience. All participants were right-handed as determined using a modified version of the Crovitz and Zener (1962) handedness questionnaire (mean score $\pm \mathrm{SD}=28.0 \pm 5.2$ ). This 18 -item inventory has scores ranging from 18 to 90 for extreme right-handers and extreme lefthanders, respectively (Crovitz and Zener, 1962). Males and females were matched according to age [two-sample $t$-test, $t(12)=-0.1$, $p=0.9]$, education level $[t(12)=1.5, p=0.2]$ and handedness $[t(12)=0.1, p=0.9]$, and had no history of neurological disease, head injury, or hearing impairment. All participants gave their informed consent. Ethical approval was granted by the Montreal Neurological Institute Ethics Review Board. TMS sessions were performed according to the published safety guidelines (Wassermann et al., 1996; Rossi et al., 2009).

\section{TASK DESCRIPTION}

Stimuli consisted of unfamiliar melodies in the western major scale, of five 0.32 -s notes in duration (Foster and Zatorre, 2010). The five-note melodies were played with low-pass-filtered harmonic tones, using pitches between $\mathrm{C} 4$ and E6. Individual trials consisted of one pair of melodies (1.6 s each) separated by $1 \mathrm{~s}$; subjects judged whether the two melodies were the same or different and indicated their response with the left or right button (see example of stimuli in Figure 1A).

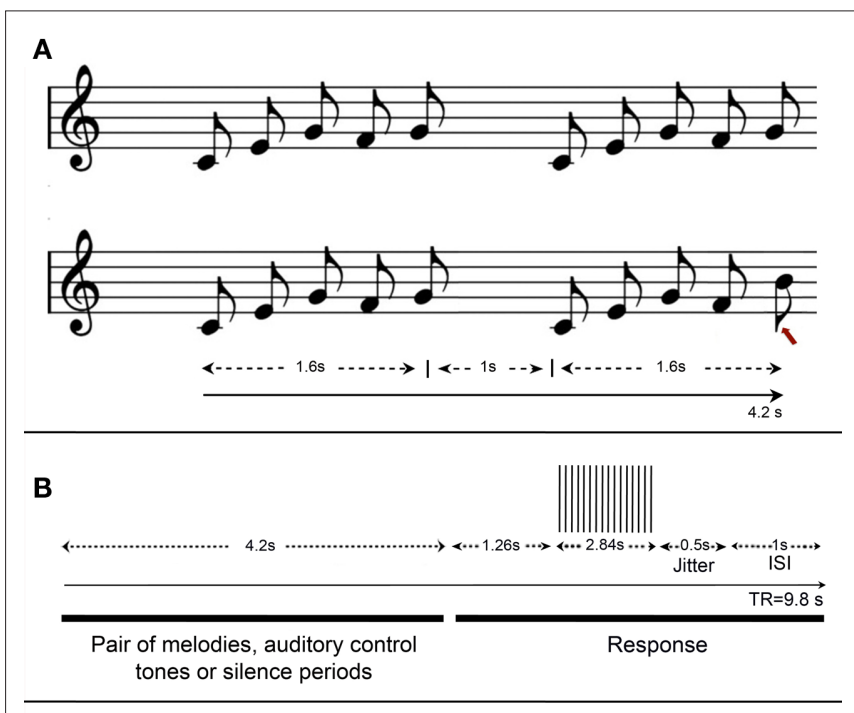

C 1Hz-rTMS
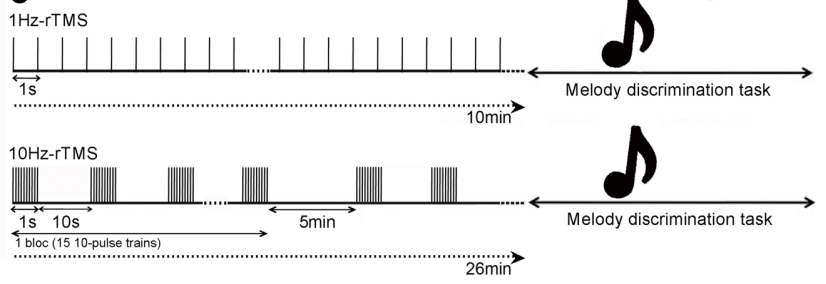

FIGURE 1 | (A) Task design. Example of auditory stimuli in the melody discrimination task. Each melody was composed of five $0.32 \mathrm{~s}$-notes lasting $1.6 \mathrm{~s}(0.32 \times 5)$, and each trial consisted of pairs of melodies separated by $1 \mathrm{~s}$, lasting $4.2 \mathrm{~s}$ in total (i.e., $1.6+1.6+1$ ). (Top) Example of a trial composed of two identical melodies; (bottom) example of a trial composed of two different melodies. The red arrow indicates melody alteration that is a difference in pitch occurring in one of the two final tones of the sequence. Participants had to indicate if the pairs of melodies were the same or different by pressing left or right buttons respectively. (B) Design for the fMRI experiment. Timeline of events during each $\mathrm{fMRI}$ trial. Each pair of melodies (or auditory control tones or silence periods) lasted $4.2 \mathrm{~s}$ and was followed by a constant $5.6 \mathrm{~s}$ including $1.26 \mathrm{~s}$ of silence, $2.84 \mathrm{~s}$ of data acquisition, $1 \mathrm{~s}$ of ISI, and a 0.5 -s jitter was added to each stimulus duration to maximize the detection of the BOLD response to the task. (C) Design for the TMS experiment. Frequencies at 1 or $10 \mathrm{~Hz}-\mathrm{rTMS}$ were applied off-line, i.e., preceding the melody discrimination task. (Top) $1 \mathrm{~Hz}$-rTMS was applied during $10 \mathrm{~min}$ (600 pulses), and (bottom) $10 \mathrm{~Hz}$-rTMS was applied in four blocks of 150 pulses each (i.e., 15 trains of 10-pulse of 1-s duration), totalizing also 600 pulses. 
Members of each melody pair were identical in terms of the duration and rhythm of tones, and differed in pitch in one of the two final tones of the sequence, which was changed by up to \pm 5 semitones (median of 2 semitones). The change maintained the key of the melody as well as the melodic contour (the order of upward and downward pitch movement in a melody without regard to magnitude).

\section{MAGNETIC RESONANCE IMAGING Anatomical MRI acquisition}

A 1-mm $\times 1-\mathrm{mm} \times 1-\mathrm{mm}$ high-resolution T1-weighted 3D image was acquired for each participant on a 3-T Siemens Trio scanner with a 32-channel Head Coil using a gradient-echo sequence (TR/ $\mathrm{TE}=2300 / 2.98,192$ slices, matrix $=256 \times 256)$.

\section{Functional MRI acquisition}

A gradient-echo EPI pulse sequence was acquired in an interleaved order to measure brain oxygenation level-dependent (BOLD) signal (3.3 $\mathrm{mm}$ isotropic voxels, 48 slices, and a $64 \times 64$ matrix size). We used a sparse-sampling paradigm (repetition time $=9.8 \mathrm{~s}$ ) to minimize any BOLD effect or auditory masking due to MRI scanning noise (Belin et al., 1999; Hall et al., 1999). Auditory events were synchronized with event-related functional magnetic resonance image volume acquisitions using Presentation software (Neurobehavioral Systems $)^{1}$. A 0.5-s jitter was added to each stimulus duration to maximize the detection of the hemodynamic responses to the task. A non-discrimination auditory control task was also included, in which subjects heard two equal-length patterns of five 0.32 -s notes, all at the same pitch of C5 and were instructed to click the left button following the second stimulus. In addition, periods of silence were inserted randomly among the task trials in each run. In total, 72 trials were presented randomly to the participants: 24 trials of melody discrimination, 24 trials of auditory control task, and 24 periods of silence, for a total duration of $12 \mathrm{~min} 16 \mathrm{~s}$. A diagram of the stimulus and scan timing is found in Figure 1B. The interstimulus interval (ISI) was $1 \mathrm{~s}$.

Our intent was to image brain activity associated with listening, comparing, and - in "different" trials - detecting differences between the two stimuli in each trial. The timing of stimuli in our experiment ensured that each fMRI scan was most sensitive to the BOLD response during the beginning and middle of the second pattern, assuming a 6- to 8-s delay to the hemodynamic response function peak (Belin et al., 1999; Hall et al., 2000). Subjects had a fixed amount of time (5.6 s) to make their response before the next trial began. They received no feedback about their responses.

In the first session (fMRI localizer), participants first completed a practice run of the melody task outside of the scanner and then another run during an fMRI scan. In a pilot study, we confirmed that one run of the melody task was sufficient to minimize further improvements in RT (unpublished results).

\section{Functional MRI data analyses}

Functional MRI data were analyzed using tools from the FMRIB Software Library ${ }^{2}$. At the first level (within-subjects), preprocessing involved several stages. The first EPI volume was deleted owing to

${ }^{1}$ www.neurobs.com/

${ }^{2}$ www.fmrib.ox.ac.uk/fsl tissue relaxation artifacts. Motion was corrected using MCFLIRT (Jenkinson and Smith, 2001). The program produced six motioncorrection parameters that were used as regressors in the design matrix. Non-brain structures were removed using BET (Smith, 2002). The data were spatially smoothed using a 5-mm Gaussian kernel of full-width at half maximum. Each dataset was normalized by a single scaling factor ("grand mean scaling"), whereby each volume in a $4 \mathrm{D}$ dataset is normalized by the same value, to allow for cross-subject statistics to be valid. High-pass temporal filtering with a 100 -s cut-off was used to remove low-frequency drifts. The resulting denoised time series data were analyzed using a general linear model (GLM) approach. Registration to MNI152 standard space was carried out using FLIRT (Jenkinson and Smith, 2001). Because of the long repetition times of the sparse-sampling paradigm, corrections for serial correlations and low-pass-filtering of the time series were not applied. Auditory trials (melody task and control task) were modeled as a single factor of interest, and the estimated motion parameters for each subject were included as covariates of no interest to reduce spurious activations because of head motion, thereby increasing statistical sensitivity. Areas of significant $\mathrm{fMRI}$ response were determined using clusters identified by a $z>2.3$ threshold and a corrected cluster threshold of $p=0.05$ assuming a Gaussian random field for the $Z$-statistics (Worsley et al., 1992; Friston et al., 1994; Forman et al., 1995).

\section{DETERMINATION OF THE TMS SITES}

Images of parameter estimates for the contrast of interest were created for each participant for the determination of the areas where the discrimination of the melodies elicited stronger task-related response than the auditory control tones. The TMS targets for the left and right HG were individually defined (in native space) by spherical regions of interest (ROI) of radius $5 \mathrm{~mm}$ (HG ROI) centered at the center of gravity (CoG) of fMRI activation clusters within the mask of HG provided by the Harvard-Oxford structural atlases $^{3}$. In case of two contiguous clusters, we chose the CoG within the cluster with the highest $z$-value. We chose the CoG instead of the peak of activation as it avoids isolated voxels and provides higher spatial resolution (Boroojerdi et al., 1999). The vertex was defined anatomically and was chosen as a control site to control for nonspecific effects of TMS such as acoustic and somatosensory artifacts.

\section{Within and between-group analysis of task-related fMRI response in the whole-brain}

Individual functional maps were transformed to MNI152 standard space to enable group comparison. To examine whether regions showed sex-related functional differences during the melody discrimination task, within and between-group comparisons were determined by examining the interaction between sex and taskrelated fMRI response. Statistical analyses were conducted with FSL using mixed-effects models to compute group differences (clustercorrected $p<0.05, z>2.3$ for within-group maps, and clustercorrected $p<0.05, z>1.6$ for between-group maps). Higher-level analysis was carried out using FLAME (FMRIB's Local Analysis of Mixed-Effects; Behrens et al., 2003). The percent of BOLD SignalChange (\%BSC) was also measured for each participant within

${ }^{3}$ http://www.fmrib.ox.ac.uk/fsl/data/atlas-descriptions.html\#ho 
the 5-mm-ROI in the left and right HG (as defined for the TMS targets, see section above). Percent of BSC values within those HG ROI were averaged across group, and the mean values were contrasted between hemispheres and between sex using one-way ANOVAs tests.

\section{Functional connectivity analysis}

Functional connectivity was conducted on the fMRI data both within and between groups using the right $\mathrm{HG}$ as seed ROI. In the within-group analysis, whole-brain maps reflecting correlations between the BOLD time course from the right HG ROI (as defined for the TMS targets) and the BOLD time course from all of the other voxels in the brain were generated for each group as follows. First, the GLM was fitted to account for the neural activity due to the discrimination melody task versus the auditory control task. Then, mean percent BOLD signal-change values were extracted from the seed HG ROI (right) and were correlated with the activity within the rest of the brain to determine where activity significantly covaried with the activity in that seed ROI (Worsley et al., 2005; cluster-corrected $p<0.05, z>2.3$ ). We then performed a between-group analysis (males versus females) by comparing the functional connectivity maps that were generated for each group (cluster-corrected $p<0.05, z>1.6$ ).

\section{TRANSCRANIAL MAGNETIC STIMULATION}

The volunteers participated in four sessions over four separate days (one session per day). After the fMRI localiser session (day 1), the volunteers participated in three TMS day sessions in which stimulation targeted the left $\mathrm{HG}$ (lHG), the right $\mathrm{HG}$ ( $\mathrm{rHG}$ ), or the Vertex (one site per day, see details here below). The sites of the stimulation (i.e., lHG, rHG, Vertex) were counterbalanced across participants. A real-time optically tracked frameless stereotaxic system (Brainsight ${ }^{\mathrm{TM}}$ Frameless, Rogue Research Inc., Montreal, Canada) was used to guide the coil over the subject's scalp. Focal TMS was delivered using a Magstim Rapid2 stimulator (Magstim Ltd., Wales, UK) equipped with an air-cooled, 70-mm, figure-ofeight coil.

\section{Determination of the motor threshold}

For each TMS session, TMS intensity was set at the resting motor threshold (MT), defined as the lowest stimulus intensity able to elicit a motor-evoked potential $\mathrm{MEP}>50 \mu \mathrm{V}$ in the relaxed abductor pollicis brevis (APB) in 5 of 10 consecutive trials at intervals $>5$ s (Rothwell et al., 1999). Each TMS day session consisted of two or four rTMS sequences, depending on which site was stimulated (see details below); each rTMS sequence was applied off-line (i.e., preceding the task), and directly followed by the melody task, see Figure 1C. The same melody discrimination task as the one described in the fMRI acquisition was used in the rTMS sessions and was presented through insert earphones (Etymotic Research, Elk Grove Village, IL, USA).

Repetitive TMS sessions targeting the lHG or rHG consisted of four rTMS sequences in which we tested two different frequencies of stimulation: 1, $10 \mathrm{~Hz}$ (see details below) and two different coil orientations: with the coil handle pointing backward and parallel to the midline (Bueti et al., 2008), or the coil handle pointing upward and perpendicular to the midline (Frank et al., 2010). When
rTMS was applied over the Vertex, a total of two rTMS sequences were carried out, one using $1 \mathrm{~Hz}-\mathrm{rTMS}$ and the other one using $10 \mathrm{~Hz}-\mathrm{rTMS}$.

- In the 1-Hz-rTMS sequence, continuous pulses at $1 \mathrm{~Hz}$ (i.e., 1 pulse per second) were applied during $10 \mathrm{~min}$ (i.e., 600 pulses). Similar protocol was used in our previous work (Andoh et al., 2006, 2008).

- In the 10-Hz-rTMS sequence, four blocks of rTMS were delivered, each block separated by a 5 -min interval. Each block consisted of 15 trains of 10-pulse of 1 -s duration (i.e., $10 \mathrm{~Hz}$ ) with an inter-train interval of $10 \mathrm{~s}$. A total of 600 pulses were delivered over a period of $26 \mathrm{~min}$; a comparable protocol has been used in previous studies (Strafella et al., 2001; Barrett et al., 2004; Andoh and Paus, 2011).

Each rTMS sequence (i.e., 1, $10 \mathrm{~Hz}$ ) was followed immediately by the auditory task. For each participant, a total of four conditions were examined [i.e., (1) $1 \mathrm{~Hz}$ frequency, coil orientation parallel to midline; (2) $1 \mathrm{~Hz}$ frequency, coil orientation perpendicular to midline; (3) $10 \mathrm{~Hz}$ frequency, coil orientation parallel to midline; (4) $10 \mathrm{~Hz}$ frequency, coil orientation perpendicular to midline], with 96 trials for each condition lasting $11 \mathrm{~min}$ each. Each rTMS sequence-auditory task was followed by a 30 -min rest interval to avoid carry-over effects from one rTMS session to the next (Rossi et al., 2000; Knecht et al., 2003). We also examined the changes in mean RT over time by dividing the trials into two 5.5-min blocks. Sites (i.e., Vertex, $r H G, l H G$ ) and the order of the sequences were counterbalanced across participants and across sex.

\section{Statistical analysis of behavioral data}

Response times were recorded from the onset of the second melody, and trials with RT beyond two SD from the mean were excluded [2294 of 21504 trials (10.7\%)]. Statistical analyses were performed using JMP software (SAS Institute, USA). The behavioral dependent variables (mean RT and accuracy) were analyzed using repeatedmeasures ANOVA with Sex (male, female) as between-subject factor and four within-subject factors: TMS Frequency $(1,10 \mathrm{~Hz})$, TMS coil Orientation (parallel, perpendicular to midline), stimulated Sites (Vertex, lHG, rHG), and Time post-TMS (Time 1: 0-5.5 min, Time 2: 5.5-11 min). For post hoc analysis, we carried out one-way ANOVA tests and used paired-t-tests with the error term derived from the ANOVA.

The rTMS-induced changes on RT were also expressed in \%RT change relative to baseline (i.e., Vertex). We used the \%RT change instead of the mean RT to evaluate the relative effect of rTMS, taking the baseline into account. Mean RT for correct responses per condition per subject were normalized to reflect relative changes caused by stimulation (Schluter et al., 1999; Devlin et al., 2003b; Gough et al., 2005) as follows: percentage of relative RT change $=100 \times(\mathrm{RT}$ with TMS lHG [or rHG]) - RT with TMS Vertex)/(RT with TMS Vertex). This type of analysis is commonly used with TMS to take into account individual differences in baseline RT (Schluter et al., 1999; Stewart et al., 2001).

In addition, we wished to test for relationships between the modulation of the behavioral effect of rTMS (\%RT change) and the degree of interhemispheric connectivity between left and right $\mathrm{HG}$. We carried 
out a whole-group analysis (including both females and males) of the functional connectivity maps obtained in the Section "Functional connectivity analysis" and added the \%RT change as a covariate to determine where functional connectivity significantly covaried with the \%RT change induced by rTMS (cluster-corrected $p<0.05, z>1.6$ ).

\section{RESULTS}

Two participants (one male and one female) were excluded from the study. The male participant did not complete all four sessions, and the female participant reported too much pain during rTMS targeting the left HG. Both data sets were excluded from the fMRI and TMS analyses.

\section{FUNCTIONAL IMAGING RESULTS: LOCALIZER SESSION Individual fMRI results}

The contrast resulting from the melody discrimination minus auditory control trials detected task-related fMRI response in bilateral HG for each participant (Figure 2A). The individual maps for the left and right CoG of the task-related fMRI response within $\mathrm{HG}$, which served as TMS targets were transformed in standard space (MNI 152) for group comparison (Figure 2B). The mean coordinates $( \pm \mathrm{SD})$ for the left $\mathrm{HG}$ CoG were $x=-48.3 \pm 1.9, y=-13.3 \pm 3.9, z=-1.4 \pm 3.3 ; Z>2.7$; and for the right HG: $x=50.8 \pm 2.2, y=-9.8 \pm 3.4, z=-0.5 \pm 3.6$; $Z>2.5$ ), see Table 1 .

\section{Group fMRI results}

Within-group analysis showed that both female and male groups had task-related fMRI responses bilaterally, in the superior temporal gyri (STG), HG, inferior frontal gyri (IFG), and precentral gyri (Figures 3A,B; Table 2). Between-group comparison differences of the functional maps showed that compared with males, females had higher task-related fMRI responses throughout auditory cortex: bilaterally in the STG, HG, and middle temporal gyri (MTG; Figure 3C).
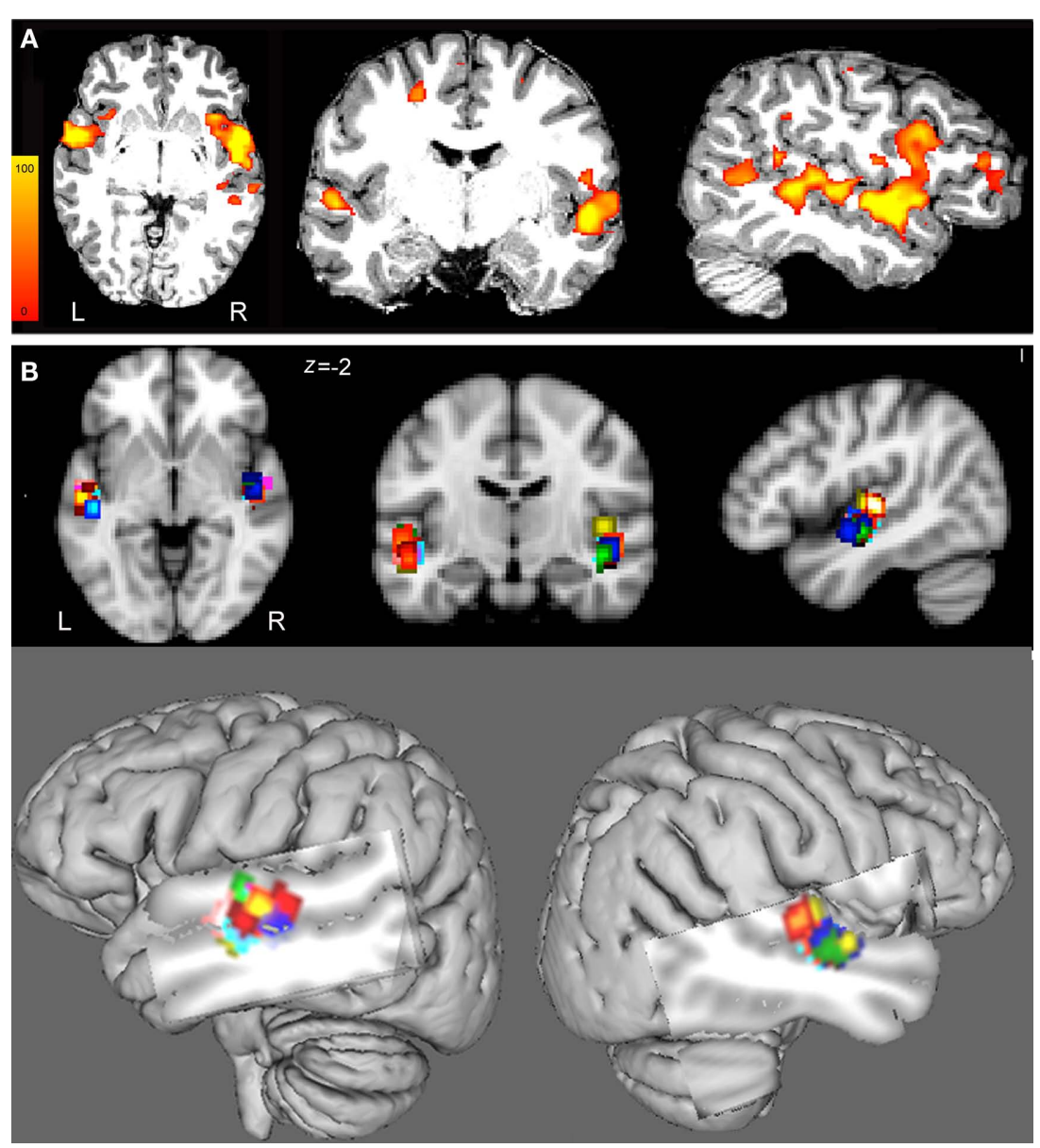

FIGURE 2 | (A) Images of parameter estimates for the contrast "melody discrimination minus auditory control tones" for a single participant. The targets of the left and right $\mathrm{HG}$ were defined individually using spherical regions of interest (radius $5 \mathrm{~mm}$ ) centered at the centers of gravity (CoG) of $\mathrm{fMRI}$ activation clusters within the mask of the Heschl's gyrus provided by the Harvard-Oxford structural atlases (http://www.fmrib.ox.ac.uk/fsl/data/atlas-descriptions. html\#ho). Z statistic images were thresholded using clusters determined by $z>2.3$ and a corrected cluster significance threshold of $p<0.05$. The individual data displayed a target for the left HG at $x=-50, y=-9, z=-3$ and for the right $\mathrm{HG}$ at $x=51, y=-6, z=-2$ in MNI 152 space. These targets were used to guide the TMS coil positioning. (B) To enable group comparison, the individual regions of interest (ROI) were transformed in MNI152 standard space (see also Table 1). (Top) From left to right: axial, coronal, and sagittal views of the 5-mm-ROI for each subject and for left and right hemispheres (each color represents one participant). (Bottom) Overlay of the 5-mm-ROI for the left and right $\mathrm{HG}$ for all participants on a $3 \mathrm{D}$ brain reconstruction. 
Table 1 | Individual coordinates $(\mathrm{mm})$ for the location of the targets for the left HG CoG (IHG ROI) and for the right HG CoG (rHG ROI) in MNI152 space and respective $z$-score values and percentage of BOLD signalchange (\%BSC) within the 5-mm HG ROI.

\begin{tabular}{|c|c|c|c|c|c|}
\hline & Subjects & $x$ & $y$ & $z$ & Z-score \\
\hline \multicolumn{6}{|c|}{ RHG ROI (MNI COORDINATES IN MM) } \\
\hline \multirow[t]{7}{*}{ Females } & $\mathrm{S} 1$ & -50 & -9 & -3 & 5.4 \\
\hline & S2 & -50 & -10 & 4 & 5.3 \\
\hline & S3 & -48 & -12 & -4 & 5.1 \\
\hline & S4 & -51 & -8 & -4 & 6.3 \\
\hline & S5 & -50 & -13 & 0 & 2.7 \\
\hline & S6 & -50 & -14 & 0 & 7.5 \\
\hline & S7 & -49 & -16 & 2 & 5.2 \\
\hline \multirow[t]{7}{*}{ Males } & S8 & -47 & -9 & -7 & 3.9 \\
\hline & S9 & -46 & -11 & -5 & 3.9 \\
\hline & S10 & -48 & -21 & 4 & 5.0 \\
\hline & S11 & -47 & -12 & -1 & 3.4 \\
\hline & $\mathrm{S} 12$ & -46 & -15 & -4 & 6.0 \\
\hline & $\mathrm{S} 13$ & -45 & -21 & -1 & 4.7 \\
\hline & S14 & -49 & -13 & -1 & 7.5 \\
\hline \multicolumn{6}{|c|}{ LHG ROI (MNI COORDINATES IN MM) } \\
\hline \multirow[t]{7}{*}{ Females } & S1 & 51 & -6 & -2 & 5.4 \\
\hline & S2 & 48 & -15 & 7 & 6.4 \\
\hline & S3 & 49 & -13 & -2 & 6.1 \\
\hline & S4 & 51 & -8 & -3 & 8.2 \\
\hline & S5 & 51 & -9 & 0 & 3.2 \\
\hline & S6 & 51 & -9 & -4 & 7.9 \\
\hline & S7 & 51 & -11 & -3 & 5.5 \\
\hline \multirow[t]{7}{*}{ Males } & S8 & 50 & -4 & -3 & 2.5 \\
\hline & S9 & 50 & -11 & -2 & 5.1 \\
\hline & $\mathrm{S} 10$ & 50 & -15 & 7 & 6.2 \\
\hline & S11 & 57 & -7 & 3 & 6.6 \\
\hline & $\mathrm{S} 12$ & 52 & -13 & 1 & 8.0 \\
\hline & $\mathrm{S} 13$ & 48 & -9 & -4 & 6.1 \\
\hline & S14 & 53 & -5 & -2 & 8.3 \\
\hline
\end{tabular}

Comparison of the percent of BOLD signal-change (\%BSC) between the left and right HG ROI showed an increase in fMRI response in the right HG ROI (mean $\pm \mathrm{SD}=0.96 \pm 0.64 \%$ ) compared with the left HG ROI (mean $\pm \mathrm{SD}=0.70 \pm 0.45 \%$ ) for both groups $[t(13)=2.2, p=0.05]$, but no differences between the two groups for the \%BSC in the left HG ROI $[t(12)=0.2, p=0.8]$, or in the right HG ROI $[t(12)=-0.4, p=0.7]$.

\section{FUNCTIONAL CONNECTIVITY ANALYSIS}

The functional connectivity analysis using the right seed HG ROI showed in the female group a correlated activity with the left HG (controlateral to the seed region), left STG, and bilaterally with the superior frontal gyrus and precentral gyrus, see Table 3; Figure 4A (left). In the male group, we found a correlated activity with the left anterior STG and posterior MTG bilaterally, see Table 3; Figure 4A (right). The functional connectivity results using the left seed HG ROI are similar to those using the right HG (data not shown).
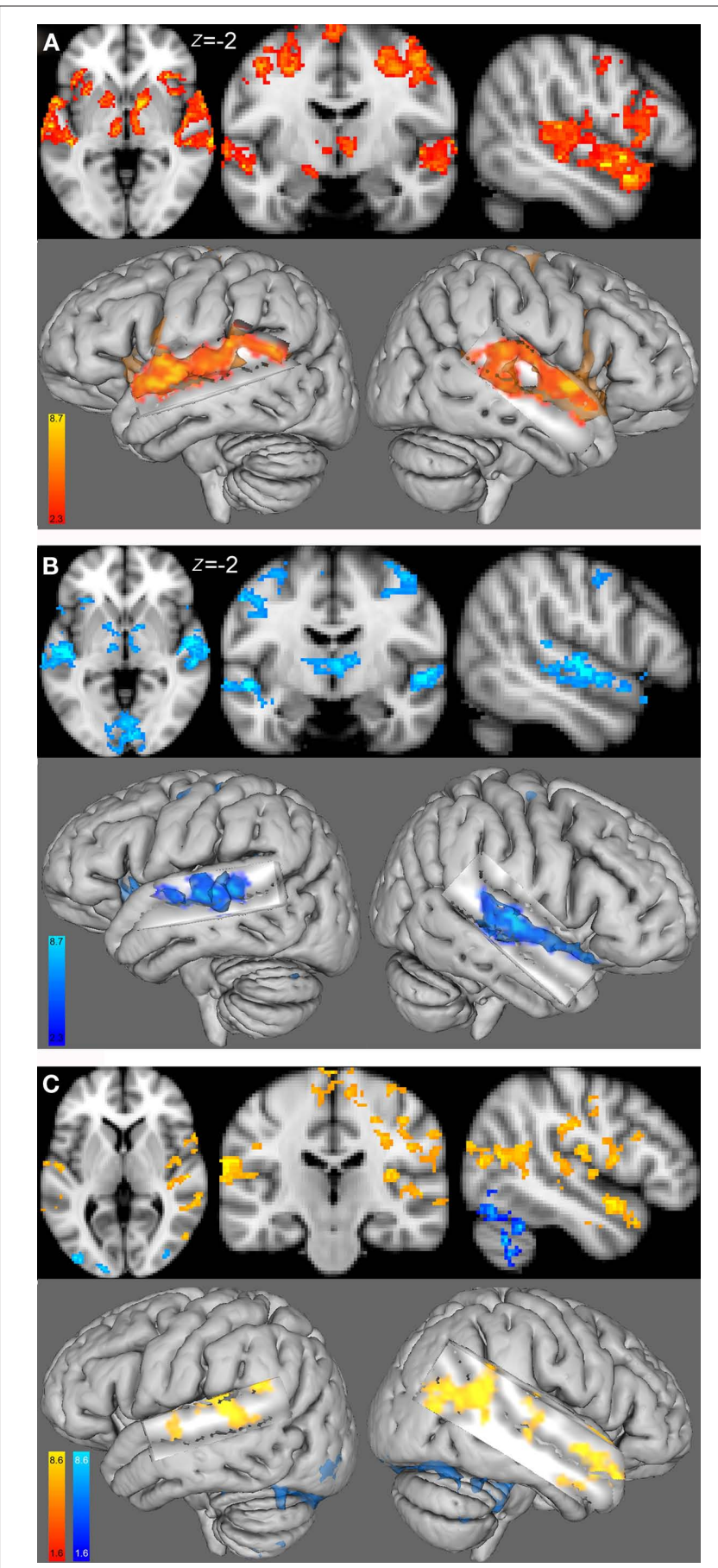

FIGURE 3 | Group functional maps for the contrast of interest, i.e., melody discrimination minus auditory control. Increased task-related activity for the contrast resulting from the melody discrimination minus auditory control trials in (A) the female group (red-yellow) and (B) the male group (blue-lightblue; cluster-corrected $p<0.05, z=2.3$ ). (C) Between-group functional maps differences $(z=4)$. In red-yellow: increased task-related activity for the females compared with males; in blue-lightblue: increased task-related activity for the males compared with females (cluster-corrected $p<0.05, z=1.6$ ).

Between-group comparison differences of the functional connectivity maps of the right HG ROI showed that compared with males, females had an increase in functional connectivity with the 
Table 2 | Mean coordinates $(\mathrm{mm})$ of the areas activated for the contrast melody discrimination minus auditory control for the female group and for the male group ( $z$-score $>2.3$, cluster-corrected threshold of $p<0.05)$.

\begin{tabular}{|c|c|c|c|c|c|}
\hline \multirow[t]{2}{*}{ Brain area } & \multicolumn{3}{|c|}{$\begin{array}{l}\text { MNI coordinates } \\
\text { in } \mathbf{~ m m}\end{array}$} & \multirow[t]{2}{*}{ Z-score } & \multirow[t]{2}{*}{$\begin{array}{l}\text { Extent } \\
\text { (voxels) }\end{array}$} \\
\hline & $x$ & $y$ & $z$ & & \\
\hline \multicolumn{6}{|l|}{ FEMALE GROUP } \\
\hline \multicolumn{6}{|l|}{ Left hemisphere } \\
\hline STG, HG & -58 & 6 & -14 & 7.35 & 2569 \\
\hline $\begin{array}{l}\text { Supplementary } \\
\text { motor cortex }\end{array}$ & -2 & 0 & 68 & 6.53 & 1638 \\
\hline Precentral gyrus & -46 & -4 & 50 & 4.47 & 610 \\
\hline IFG (POp) & -54 & 12 & 6 & 4.01 & 60 \\
\hline \multicolumn{6}{|l|}{ Right hemisphere } \\
\hline $\begin{array}{l}\text { STG, HG, } \\
\text { supramarginal gyrus }\end{array}$ & 58 & 4 & -8 & 8.66 & 3301 \\
\hline Precentral gyrus & 48 & -6 & 46 & 5.01 & 729 \\
\hline IFG (PTr) & 42 & 40 & 8 & 3.51 & 23 \\
\hline \multicolumn{6}{|l|}{ MALE GROUP } \\
\hline \multicolumn{6}{|l|}{ Left hemisphere } \\
\hline STG, HG & -44 & -22 & -2 & 5.26 & 1090 \\
\hline IFG (POp) & -48 & 12 & 4 & 3.01 & 17 \\
\hline Precentral gyrus & -54 & 0 & 42 & 4.83 & 471 \\
\hline $\begin{array}{l}\text { Inferior frontal } \\
\text { gyrus (POp) }\end{array}$ & 46 & 10 & 22 & 3.17 & 23 \\
\hline Paracingulate gyrus & -8 & 16 & 42 & 4.68 & 744 \\
\hline Frontal orbital cortex & -28 & 22 & -8 & 4.17 & 188 \\
\hline Parahippocampal gyrus & -12 & -28 & -10 & 3.1 & 8 \\
\hline Cingulate gyrus & -8 & 28 & 26 & 2.83 & 18 \\
\hline Precentral gyrus & -40 & -18 & 62 & 2.81 & 10 \\
\hline \multicolumn{6}{|l|}{ Right hemisphere } \\
\hline STG, HG & 58 & -12 & 0 & 6.92 & 735 \\
\hline IFG (PTr) & 54 & 20 & -4 & 4.32 & 74 \\
\hline MTG & 46 & -40 & 2 & 4.15 & 24 \\
\hline Precentral gyrus & 46 & -6 & 56 & 4.03 & 192 \\
\hline Frontal orbital cortex & 34 & 30 & 2 & 3.32 & 5 \\
\hline Cingulate gyrus & 2 & 14 & 20 & 3.06 & 13 \\
\hline
\end{tabular}

STG, superior temporal gyrus; MTG, middle temporal gyrus; HG, Heschl's gyrus; IFG (PTr), Inferior frontal gyrus (pars triangularis) or (POp) pars opercularis.

left HG, and bilaterally with the superior frontal gyri, and precentral gyri (see Figure 4B, left). In males compared with females, we found an increase in functional connectivity with areas in the same hemisphere as the seed region, i.e., the right anterior superior temporal gyrus, the right inferior frontal gyrus (pars orbitalis), right precentral gyrus, as well as in the contralateral hemisphere to the seed region, e.g., the left anterior superior temporal gyrus, left posterior middle temporal gyrus and left posterior inferior temporal gyrus (see Figure 4B, right).

\section{TMS RESULTS}

Motor thresholds ranged from 53 to $69 \%$ of maximum stimulator (mean: $61 \pm 6 \%$ ).

Table 3 | Mean coordinates $(\mathrm{mm}$ ) of the areas functionally interconnected with the right HG ROI during the contrast melody discrimination versus auditory control in the female group, and the male group. Only clusters with more than 10 voxels are reported.

Brain area

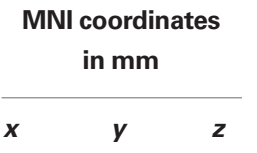

Z-scor

Cluster level

FEMALE GROUP
Left hemisphere
HG, STG
Supplementary
motor area
Postcentral gyrus
Precentral gyrus
Superior frontal gyrus
Supramarginal gyrus
Right hemisphere
Superior frontal gyrus
Supplementary
motor area
Precentral gyrus
Superior parietal
lobule
Postcentral gyrus
Parahippocampal
gyrus

(voxels)

\begin{tabular}{lrrrrr} 
MALE GROUP & & & & & \\
Left hemisphere & & & & & \\
$\quad$ Anterior STG & -44 & -8 & -10 & 6.78 & 1686 \\
$\quad$ Cingulate gyrus & -4 & 6 & 40 & 4.43 & 55 \\
$\quad$ Supplementary & -2 & 4 & 48 & 3.89 & 58 \\
$\quad$ motor cortex & & & & & \\
$\quad$ Posterior MTG & -54 & -52 & 10 & 3.34 & 21 \\
$\quad$ Parahippocampal & -26 & -16 & -24 & 3.12 & 12 \\
$\quad$ gyrus & & & & & \\
Right hemisphere & & & & & 3.15 \\
$\quad$ Posterior MTG & 58 & -36 & 0 & 4.15 & 385 \\
Inferior frontal & 42 & 20 & -8 & 3.41 & 38 \\
$\quad$ cortex (POr) & & & & & \\
Precentral gyrus & 52 & 8 & 10 & 3.7 & 14 \\
\hline
\end{tabular}

IFG (POr), Inferior frontal gyrus (pars orbitalis).

Overall accuracy in the melody task was $83 \%$ and was not affected by TMS, either at time 1, i.e., (0-5.5 min) after rTMS (Table 4) or at time 2, i.e., (5.5-11 $\mathrm{min}$ ) after rTMS (Table 4). The interaction between score, orientation, frequency, and sex was not significant (repeated-measures ANOVA $[F(1,12)<0.35 ; p=0.9]$.

Repeated-measures ANOVA carried out on mean RT showed no significant main effects of Frequency, Orientation, Time, Sex, or Sites $[F(2,12)=1.4 ; p>0.2 ; \mathrm{NS}]$ but a significant interaction between Sex and Sites $[F(2,12)=3.5, p=0.03]$, as well as a significant interaction between Frequency, Time, Sites, and Sex 

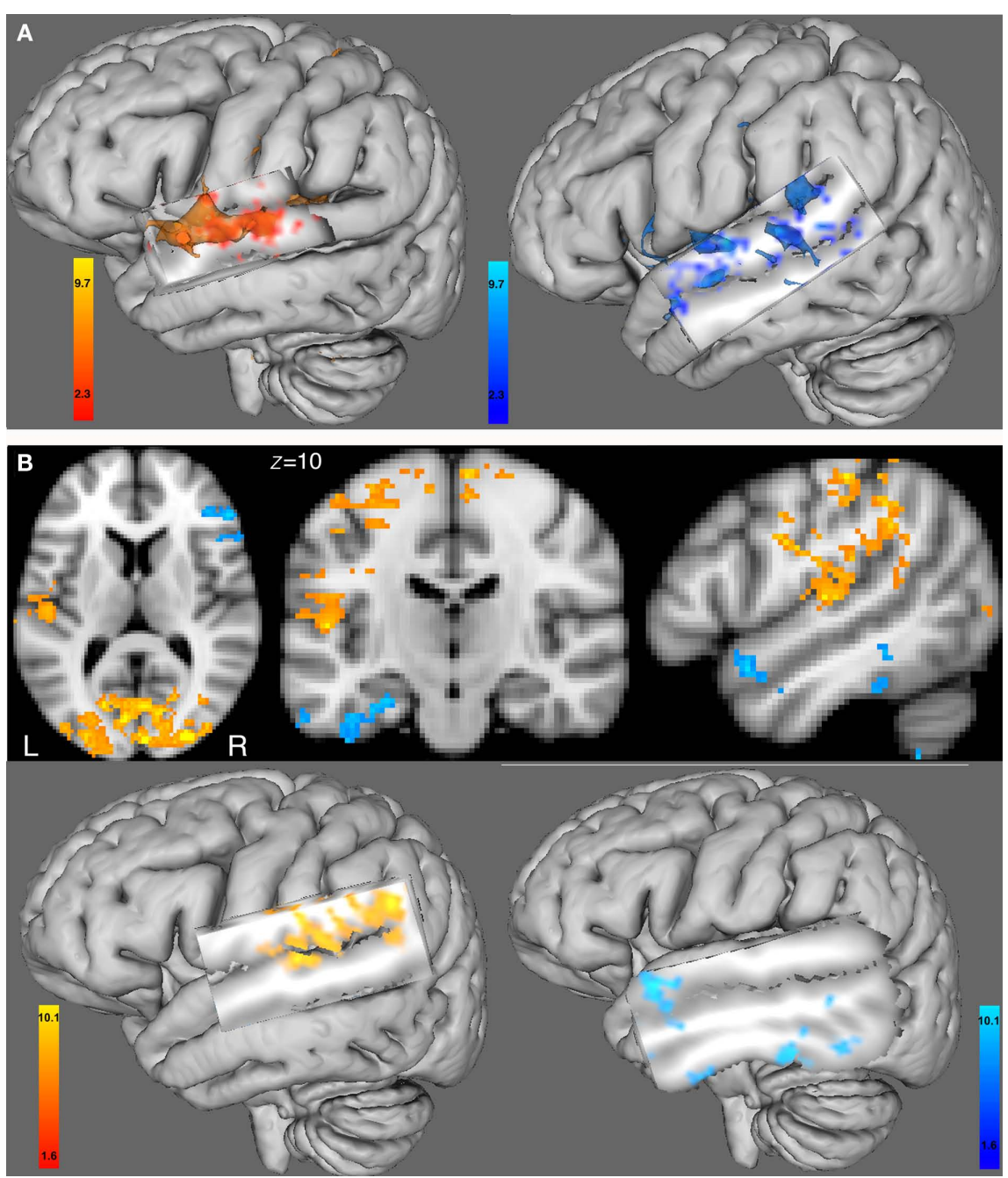

FIGURE 4 | (A) Functional connectivity with the BOLD time course from the seed region of the right HG ROI. Left hemisphere 3D view for (left) the female group and (right) the male group (cluster-corrected $p<0.05, z=2.3$ ). (B) Between-group comparison of the functional connectivity with the BOLD time course from the right $\mathrm{HG}$ ROI using 2D views (top) or 3D views (bottom). In red-yellow: increased in functional connectivity with the right HG in females compared with males; in blue-lightblue: increased in functional connectivity with the right $\mathrm{HG}$ in males compared with females (cluster-corrected $p<0.05$, $z=1.6)$. All $3 \mathrm{D}$ views were displayed by cutting sagittal slices through the left temporal cortex in order to focus on left temporal regions.
$[F(2,12)=5.5, p<0.01]$. We did not find any interaction with orientation $[F(2,12)=1.2 ; p>0.2 ; \mathrm{NS}]$, therefore, data from the two orientations were averaged for each participant.

At Time 1 (i.e., 0-5.5 min after rTMS), $10 \mathrm{~Hz}$-rTMS targeting rHG significantly decreased RT compared with $10 \mathrm{~Hz}-\mathrm{rTMS}$ applied over the vertex in the female group [paired Student's $t$-test $t(6)=-2.7, p<0.05]$ and significantly increased RT in the male group $[t(6)=2.2, p<0.02]$. However, $10 \mathrm{~Hz}$-rTMS targeting $\mathrm{lHG}$ was not significant either in the female group $[t(6)=1.2, p=0.2]$, or in the male group $[t(6)=-0.8, p=0.5]$.

Compared with $1 \mathrm{~Hz}$-rTMS applied over the vertex, no significant effect was found with $1 \mathrm{~Hz}-\mathrm{rTMS}$ targeting $\mathrm{rHG}$ in the female group $[t(6)=-0.9, p=0.3]$ or in the male group $[t(6)=-1.04$, $p=0.3$. However, $1 \mathrm{~Hz}$-rTMS targeting $\mathrm{lHG}$ was significant in the female group $[t(6)=3.2, p<0.02]$ but not in the male group $[t(6)=-0.2, p=0.9)$, Figure 5A.
At Time 2 (i.e., 5.5-11 min after rTMS), 10 Hz-rTMS targeting rHG significantly increased RT compared with $10 \mathrm{~Hz}$-rTMS applied over the vertex in the female group $[t(6)=-4.1, p<0.01]$. The other conditions did no reach significance $[t(6)=-0.83, p>0.5$; NS], Figure 5B.

To take into account individual differences in baseline RT, Figure 5 represents normalized RT and expressed in \%RT change relative to the stimulation applied over the Vertex.

\section{WHOLE-GROUP ANALYSIS OF THE FUNCTIONAL CONNECTIVITY MAPS ADDING \%RT CHANGE AS A COVARIATE}

In order to test whether there was any relationship between the pattern of TMS results and interhemispheric connectivity between left and right auditory cortices, whole-group analysis (including both females and males) of the functional connectivity maps using the right HG as a seed ROI were carried out with the \%RT change added as a covariate for time 1, i.e., (0-5.5 $\mathrm{min})$ after rTMS. 
Table 4 | Mean score (\%₫SEM) for each condition (i.e., sex, rTMS frequency, and area stimulated) at time 1 (0-5.5 $\mathrm{min}$ ) and time 2 (5.5-11 min).

\begin{tabular}{|c|c|c|c|c|}
\hline \multirow{2}{*}{$\begin{array}{l}\text { Mean score } \\
(\% \pm S E M) \text { time } 1\end{array}$} & \multicolumn{2}{|c|}{$1 \mathrm{~Hz}-\mathrm{rTMS}$} & \multicolumn{2}{|c|}{$10 \mathrm{~Hz}$-rTMS } \\
\hline & Females & Males & Females & Males \\
\hline Vertex & $82.7 \pm 3.9$ & $84.2 \pm 3.4$ & $85.7 \pm 3.1$ & $84.8 \pm 3.1$ \\
\hline Left HG & $83.6 \pm 2.9$ & $84.5 \pm 2.5$ & $84.6 \pm 2.9$ & $84.2 \pm 2.6$ \\
\hline Right HG & $81.2 \pm 2.4$ & $84.2 \pm 2.3$ & $80.9 \pm 2.0$ & $85.5 \pm 1.9$ \\
\hline \multirow{2}{*}{$\begin{array}{l}\text { Mean score } \\
(\% \pm S E M) \text { time } 2\end{array}$} & $1 \mathrm{~Hz}$ & $1 \mathrm{~Hz}$ & $10 \mathrm{~Hz}$ & $10 \mathrm{~Hz}$ \\
\hline & Females & Males & Females & Males \\
\hline Vertex & $81.2 \pm 4.1$ & $85.1 \pm 2.7$ & $80.0 \pm 2.7$ & $86.0 \pm 4.3$ \\
\hline Left HG & $81.2 \pm 3.0$ & $83.7 \pm 2.0$ & $79.3 \pm 2.9$ & $83.1 \pm 2.1$ \\
\hline Right HG & $80.6 \pm 2.6$ & $84.2 \pm 2.2$ & $80.8 \pm 3.0$ & $82.4 \pm 2.4$ \\
\hline
\end{tabular}

We found a negative correlation between the \%RT change caused by $10 \mathrm{~Hz}$-rTMS targeting rHG and the functional interhemispheric connectivity between right and left auditory cortices, including bilateral STG, bilateral MTG and the left HG (see Figure 6A, left). This result shows that the higher the functional connectivity between left and right auditory cortices, the higher the facilitation (\%RT decrease) caused by $10 \mathrm{~Hz}-\mathrm{rTMS}$ over the right HG.

Similar analysis using the \%RT change when $10 \mathrm{~Hz}$-rTMS targeted the left HG did not show any significant change in the areas of interest (see Figure 6A, right).

Similarly to $10 \mathrm{~Hz}-\mathrm{rTMS}$, we found a negative correlation between the $\%$ RT change caused by $1 \mathrm{~Hz}-\mathrm{rTMS}$ targeting $\mathrm{lHG}$ and the functional changes in interhemispheric connectivity between left and right auditory cortices, including the right STG, the right $\mathrm{HG}$, and right posterior middle temporal gyrus (see Figure 6B, left). Similar analysis using the \%RT change when $1 \mathrm{~Hz}-\mathrm{rTMS}$ targeted the right $\mathrm{HG}$ did not show any significant change in the areas of interest (see Figure 6B, right).

\section{A Time 1: [0 - $5.5 \mathrm{~min}]$}

\section{$\%$ RT change using $10 \mathrm{~Hz}-\mathrm{rTMS}$}

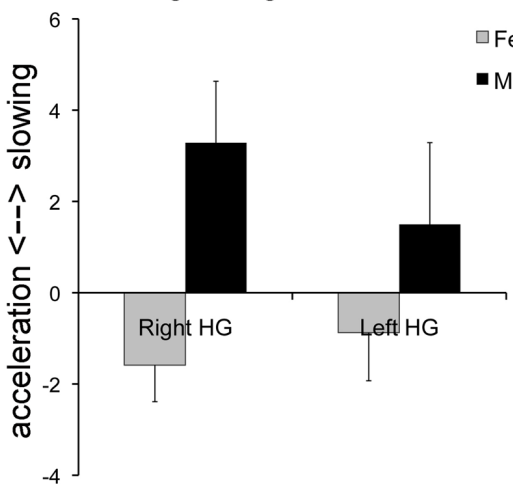

B Time 2: [5.5 - $11 \mathrm{~min}]$

$\%$ RT change using $10 \mathrm{~Hz}-\mathrm{rTMS}$

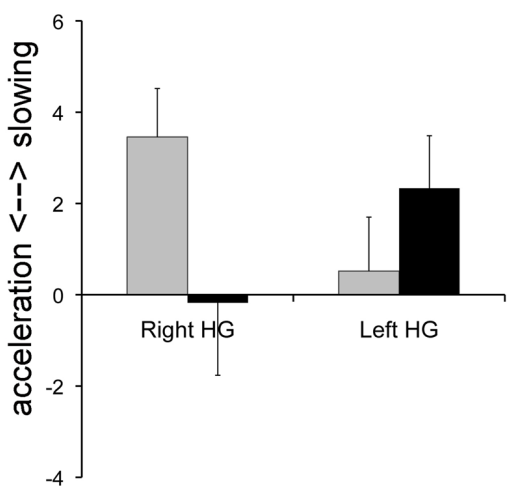

FIGURE 5 | (A) At time 1 (0-5.5 min after rTMS; left) \%RT change with $10 \mathrm{~Hz}$-rTMS targeting the right or left HG calculated relative to rTMS applied over the Vertex for the female group (black bars) and the male group (gray bars). (Right) \%RT change with $1 \mathrm{~Hz}-\mathrm{rTMS}$ targeting the right or left HG calculated relative to rTMS applied over the Vertex for the female group and the male group. (B) At time 2 (5.5-11 min after rTMS; left) \%RT change with $10 \mathrm{~Hz}-\mathrm{rTMS}$
$\%$ RT change using $1 \mathrm{~Hz}-\mathrm{rTMS}$

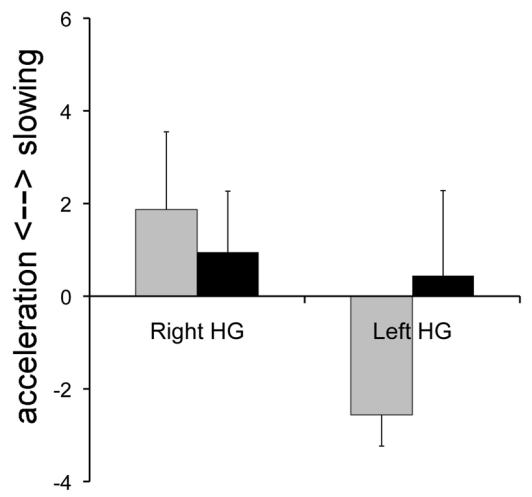

$\%$ RT change using $1 \mathrm{~Hz}-\mathrm{rTMS}$

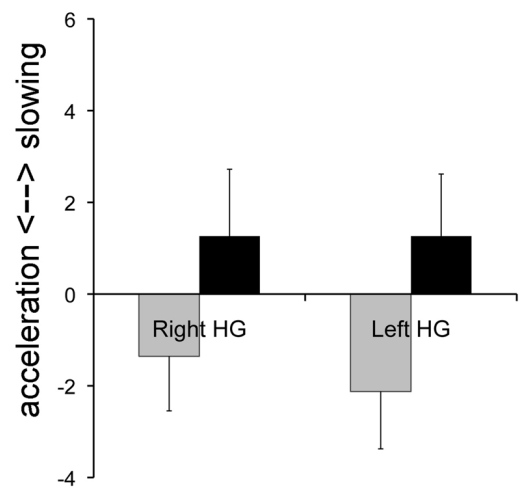

targeting the right or left HG calculated relative to rTMS applied over the Vertex for the female group (black bars) and the male group (gray bars). (Right) \%RT change with $1 \mathrm{~Hz}-\mathrm{rTMS}$ targeting the right or left $\mathrm{HG}$ calculated relative to $\mathrm{rTMS}$ applied over the Vertex for the female group and the male Error bars reflect SE of the mean adjusted to correctly reflect the variance in the within-subject design (Loftus and Masson, 1994). 

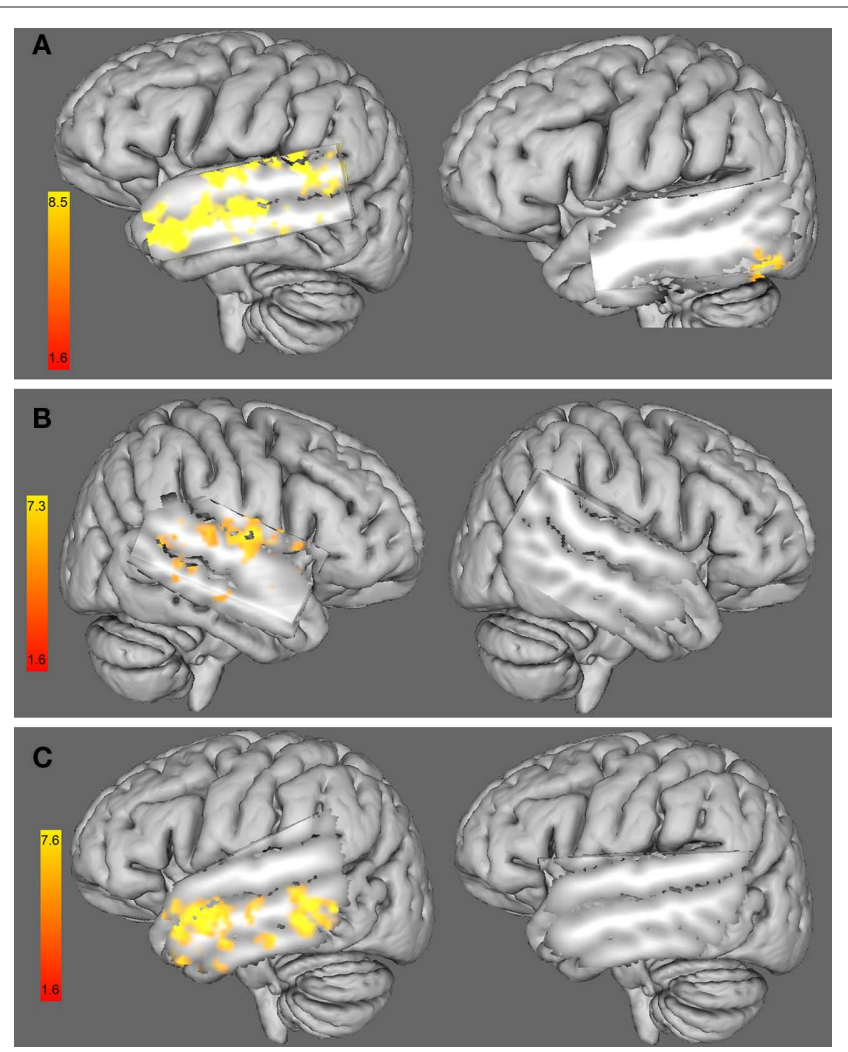

FIGURE 6 | (A) 3D left hemisphere view of a whole-group analysis (including females and males) of the functional connectivity maps using the right $\mathrm{HG}$ as a seed ROI and adding (left) the \% RT change with $10 \mathrm{~Hz}-\mathrm{rTMS}$ targeting the right $\mathrm{HG}$ at time 1 (i.e., 0-5.5 min) as a covariate and (right) the \% RT change with $10 \mathrm{~Hz}-\mathrm{rTMS}$ targeting the left $\mathrm{HG}$ at time 1 (i.e., 0-5.5 min) as a covariate. (B). Three dimensional right hemisphere view of a whole-group analysis (including female and male) of the functional connectivity maps using the left $\mathrm{HG}$ as a seed ROI and adding (left) the \%RT change when $1 \mathrm{~Hz}-\mathrm{rTMS}$ targeted the left $\mathrm{HG}$ at time 1 (i.e., 0-5.5 min) as a covariate and (right) the \% RT change when $1 \mathrm{~Hz}$-rTMS targeted the right $\mathrm{HG}$ at time 1 (i.e., 0-5.5 min) as a covariate. (C) 3D left hemisphere view of a whole-group analysis (including female and male) of the functional connectivity maps using the right $\mathrm{HG}$ as a seed $\mathrm{ROI}$ and adding (left) the \%RT change when $10 \mathrm{~Hz}$-rTMS targeted the right $\mathrm{HG}$ at time 2 (i.e., 5.5-11 $\mathrm{min}$ ) as a covariate and (right) the \% RT change when $10 \mathrm{~Hz}-\mathrm{rTMS}$ targeted the left $\mathrm{HG}$ at time 2 (i.e., 5.5-11 min) as a covariate. Three dimensional views were displayed by cutting sagittal slices through the left temporal cortex in order to focus on left temporal regions.

A similar analysis was carried out to look at the correlation between functional connectivity and the \%RT change at time 2, i.e., (5.5$11 \mathrm{~min}$ ) after $10 \mathrm{~Hz}$-rTMS targeting the right $\mathrm{HG}$. We found a negative correlation between the \%RT change after 5.5 min of $10 \mathrm{~Hz}-\mathrm{rTMS}$ targeting the right $\mathrm{HG}$ and the \%BSC in contralateral areas to the stimulated site, such as the left middle and inferior temporal gyri but not the auditory cortex (Figure 6C, left). Similar analysis using the \%RT change when $10 \mathrm{~Hz}$-rTMS targeted the left HG did not show any significant change in the areas of interest (see Figure 6C, right).

\section{DISCUSSION}

Using a melody discrimination task we found differential effects on RT after $10 \mathrm{~Hz}$-rTMS targeting the right HG which interacted with sex; that is, compared with $10 \mathrm{~Hz}$-rTMS applied over the Vertex,
$10 \mathrm{~Hz}$-rTMS targeting the right HG decreased RT (improved performance) in the female group but increased RT (worsened performance) in the male group.

No significant effect of $10 \mathrm{~Hz}$-rTMS targeting the left HG was found either for the female or the male group. The rTMS-induced modulation on the right $\mathrm{HG}$ is consistent with the crucial role of the right HG in melody processing that has been reported in healthy subjects using imaging techniques (Zatorre and Belin, 2001b; Zatorre and Gandour, 2008), as well as with lesion studies showing that patients with a right temporal lobectomy were impaired at melody tasks (Stewart et al., 2006). This finding is also consistent with a recent study using transcranial direct current stimulation (tDCS) on auditory cortices, showing that tDCS applied over the right HG interfered with pitch discrimination task more than tDCS applied over the left HG (Mathys et al., 2010). However, their procedures were not as specific as ours, indeed we used individually defined targets of the left and right HG based on both anatomical and functional criteria using fMRI during an auditory task of melody discrimination and rTMS was applied off-line, i.e., prior to the auditory task of melody discrimination.

We also found RT changes depending on the rTMS frequency. Compared with $1 \mathrm{~Hz}-\mathrm{rTMS}$ applied over the vertex, females had a significant RT decrease using $1 \mathrm{~Hz}$-rTMS targeting the left HG, but males did not. This RT decrease was higher than the one found using $10 \mathrm{~Hz}$-rTMS targeting the right $\mathrm{HG}(-2.6 \pm 0.7$ and $-1.6 \pm 0.8 \%$ respectively), which is consistent with previous data (Andoh et al., 2008). Similarly to $10 \mathrm{~Hz}-\mathrm{rTMS}$, the percentage of relative RT change induced by $1 \mathrm{~Hz}-\mathrm{rTMS}$ targeting the left HG was negatively correlated with the functional connectivity between left and right auditory cortices, indicating that across individuals the higher the facilitation (RT decrease), the higher the interhemispheric connectivity between auditory cortices. In motor cortex studies, low-frequency rTMS applied over the left motor cortex has been shown to reduce interhemispherical inhibition of the right motor cortex resulting in an increase in corticospinal excitability in the right motor cortex (Gilio et al., 2003; Schambra et al., 2003). Similarly, we suggest that $1 \mathrm{~Hz}-\mathrm{rTMS}$ applied over the left HG could increase neuronal excitability in the right HG perhaps through transcallosal disinhibition mechanisms, and because of the crucial role of the right HG in melody processing, this would lead to a facilitating of performance at the melody task. In contrast, no effect of $1 \mathrm{~Hz}$-rTMS applied over the left HG was found in males, which is not surprising, considering the relatively lower communication between left and right HG, suggesting that in males, right HG function is preserved after rTMS-induced interference over the left HG. This frequency-dependent change in RT could be due to compensatory mechanisms that adapt the auditory network to changes in cortical excitability induced by the different frequencies of rTMS (Pascual-Leone et al., 1998; Strafella and Paus, 2001; Rounis et al., 2005; Andoh et al., 2008; Hoogendam et al., 2010).

In addition, we found an effect of time after $10 \mathrm{~Hz}$-rTMS targeting the right $\mathrm{HG}$ for the female group only, starting with a significant RT decrease during the first $5.5 \mathrm{~min}$, and then a RT increase for the last $5.5 \mathrm{~min}$. The RT increase for the last $5.5 \mathrm{~min}$ was negatively correlated with the \%BSC in the left middle and inferior temporal 
gyri, which could indicate a spread of neuronal excitability from left superior temporal regions to inferior temporal regions, thus perhaps slowing the auditory processing and therefore impairing melody performance. For all other conditions, we did not find a change in RT over time, suggesting that the functional disruption caused by rTMS recovered quickly. The physiological basis of rTMS-induced effects on the brain is still undefined, and the mechanisms underlying the changes in excitability and the duration vary, depending on the circuits in which they operate (Hoogendam et al., 2010).

We did not find an effect of coil orientation when comparing two different orientations, with the coil handle placed approximatively parallel or perpendicular to the midline (Bueti et al., 2008; Frank et al., 2010). Previous motor cortex studies showed that optimal TMS response is obtained with current flow perpendicular to the central sulcus (Mills et al., 1992; Rothwell et al., 1992), thus suggesting differences in cortical folding between motor and non-motor areas, which could therefore affect current direction. Further studies using diffusion tensor MRI scans might be useful for assessing the orientation of the white matter fibers in HG (Herbsman et al., 2009).

The fMRI results showed a functional asymmetry in auditory melody processing with a higher task-related activity in the right auditory cortex compared with the left, which is consistent with the previous literature (Zatorre and Belin, 2001a; Patterson et al., 2002; Zatorre et al., 2002; Hyde et al., 2008). We also found sex differences in task-related response in left and right auditory cortices such that there was a higher functional connectivity between left and right auditory cortices in female compared with male individuals. A similar finding has already been reported in language studies (Schmithorst and Holland, 2007; Bitan et al., 2010). This sex-related difference in functional connectivity could be very relevant to understand rTMSinduced effects. Indeed, when we look at the correlation maps between the patterns of functional connectivity and the \%RT change induced by rTMS (Figure 6), we do see a relationship between the two, indicating that across individuals the higher the facilitation (RT decrease), the higher the interhemispheric connectivity between auditory cortices. In addition, the correlation maps between the patterns of functional connectivity and the \%RT change induced by rTMS were consistent across rTMS frequencies (i.e., $1,10 \mathrm{~Hz}$ ), demonstrating that functional connectivity maps can help to understand TMS-induced effects. This is the first study reporting a relationship between rTMSinduced behavioral changes and interhemispheric functional connectivity, but we suggest that understanding such relationships may prove critical in the long run to interpreting the effects of rTMS on lateralized functions.

In the female group, it seems that the facilitatory effect we observed could be due to the modulation of the functional connectivity induced by rTMS between left and right HG. This conclusion is based on the following observations: (1) the significant RT decrease after $10 \mathrm{~Hz}$-rTMS targeting the right HG; (2) the high functional connectivity between right and left auditory cortices; and (3) the correlation between the \%RT change when $10 \mathrm{~Hz}-\mathrm{rTMS}$ targeted the right HG and the functional connectivity between right and left auditory cortices. Indeed, the relatively higher communication between left and right auditory cortices in women could suggest that females compensate with their left HG after rTMS-induced interference on the right $\mathrm{HG}$.

In the male group, if we consider (1) the significant RT increase after $10 \mathrm{~Hz}$-rTMS targeting the right HG; and (2) the low functional connectivity between right and left auditory cortices, we suggest that the low communication between right and left HG may explain why males are not able to compensate with their left HG after rTMS-induced interference on the right HG, by comparison with females. These findings are in line with lesion studies (Thiel et al., 2006a,b) and with previous TMS work (Andoh and Martinot, 2008; Andoh and Paus, 2011) showing that some brain functions may operate in a state of interhemispheric compensation after a permanent or temporary disruption. It has been shown that the homologous area in the contralateral hemisphere can play a crucial role in the recovery process, maybe through a mechanism of reduced transcallosal disinhibition (Thiel et al., 2006b; O'shea et al., 2007). And because of the high level of communication between both left and right HG in the female group, it seems possible that after $r T M S$-induced interference in the right $\mathrm{HG}$, the left $\mathrm{HG}$ could play a compensatory role to enable the execution of the melody task.

\section{LIMITATIONS}

We reported a relationship between the functional connectivity between left and right HG and the \%RT change after off-line rTMS. However, our sample is relatively small, even if comparable to previous studies (Devlin et al., 2003a; Gilio et al., 2003; Gough et al., 2005) and further studies combining TMS and brain imaging (i.e., during TMS or pre- and post-TMS) are needed to address this important question in detail.

Another limiting factor of this study may be related to the depth of the stimulated areas. The electric field strength is maximal up to $2 \mathrm{~cm}$ away from the coil and drops off exponentially in depth (Maccabee et al., 1990; Zangen et al., 2005). The average depth of the HG targets from the center of the coil was inferior to $3 \mathrm{~cm}$; however, depth was not significantly different between the groups, and therefore could not explain the differential effect of TMS response. Similar depth from the center of the TMS coil has been reported for some participants using rTMS applied over the motor cortex (Stokes et al., 2005). It is unlikely that our procedures resulted in rTMS-induced changes in the medial portion of $\mathrm{HG}$, the site of primary auditory cortex; conversely, HG extends all the way to the lateral edge of the superior temporal gyrus, and this area, which is thought to play a role in pitch processing (Johnsrude et al., 2000; Puschmann et al., 2010) was very likely targeted by TMS.

We cannot also preclude that while targeting $\mathrm{HG}$, we could have stimulated surrounding superior temporal regions, including adjacent parabelt regions of the auditory cortex (Penhune et al., 1996; Morosan et al., 2001), which have been shown to play a role in processing of music, and therefore could have interfered with the present melody discrimination task (Penhune et al., 1996; Morosan et al., 2001; Foster and Zatorre, 2010).

\section{CONCLUSION}

Here we report a relationship between the behavioral modulation induced by rTMS and the pattern of interhemispheric functional connectivity as measured via fMRI during melody 
processing. The interaction between sex and patterns of rTMSinduced changes in RT that we observed may thus be better understood in terms of differential patterns of interhemispheric communication in males and females. Such findings might help to understand the differential effects in behavior reported in previous rTMS studies and deserve further investigation.

\section{REFERENCES}

Andoh, J., Artiges, E., Pallier, C., Riviere, D., Mangin, J. F., Cachia, A., Plaze, M., Paillere-Martinot, M. L., and Martinot, J. L. (2006). Modulation of language areas with functional MR image-guided magnetic stimulation. Neuroimage 29, 619-627.

Andoh, J., Artiges, E., Pallier, C., Riviere, D., Mangin, J. F., Paillere-Martinot, M. L., and Martinot, J. L. (2008). Priming frequencies of transcranial magnetic stimulation over Wernicke's area modulate word detection. Cereb. Cortex 18, 210-216.

Andoh, J., and Martinot, J. L. (2008). Interhemispheric compensation: a hypothesis of TMS-induced effects on language-related areas. Eur. Psychiatry 23, 281-288.

Andoh, J., and Paus, T. (2011). Combining functional neuroimaging with off-line brain stimulation: modulation of task-related activity in language areas. J. Cogn. Neurosci. 23, 349-361.

Barrett, J., Della-Maggiore, V., Chouinard, P. A., and Paus, T. (2004). Mechanisms of action underlying the effect of repetitive transcranial magnetic stimulation on mood: behavioral and brain imaging studies. Neuropsychopharmacology 29, 1172-1189.

Behrens, T., Rohr, K., and Stiehl, H. S. (2003). Robust segmentation of tubular structures in 3-D medical images by parametric object detection and tracking. IEEE Trans. Syst. Man Cybern. B Cybern. 33, 554-561.

Belin, P., Zatorre, R. J., Hoge, R., Evans, A. C., and Pike, B. (1999). Eventrelated fMRI of the auditory cortex. Neuroimage 10, 417-429.

Berardelli, A., Inghilleri, M., Rothwell, J. C., Romeo, S., Curra, A., Gilio, F., Modugno, N., and Manfredi, M. (1998). Facilitation of muscle evoked responses after repetitive cortical stimulation in man. Exp. Brain Res. 122, 79-84.

Bitan, T., Lifshitz, A., Breznitz, Z., and Booth, J. R. (2010). Bidirectional connectivity between hemispheres occurs at multiple levels in language processing but depends on sex. J. Neurosci. 30, 11576-11585.

Boemio, A., Fromm, S., Braun, A., and Poeppel, D. (2005). Hierarchical and asymmetric temporal sensitivity in human auditory cortices. Nat. Neurosci. 8, 389-395.

Boroojerdi, B., Foltys, H., Krings, T., Spetzger, U., Thron, A., and Topper, R. (1999). Localization of the motor hand area using transcranial magnetic stimulation and functional magnetic resonance imaging. Clin. Neurophysiol. 110, 699-704.

Bueti, D., van Dongen, E. V., and Walsh, V. (2008). The role of superior temporal cortex in auditory timing. PLoS ONE 3, e2481. doi: 10.1371/journal. pone. 0002481

Chen, R., Classen, J., Gerloff, C., Celnik, P., Wassermann, E. M., Hallett, M., and Cohen, L. G. (1997). Depression of motor cortex excitability by lowfrequency transcranial magnetic stimulation. Neurology 48, 1398-1403.

Crovitz, H. F., and Zener, K. (1962). A group-test for assessing hand- and eye-dominance. Am. J. Psychol. 75, 271-276.

Devlin, J. T., Matthews, P. M., and Rushworth, M. F. (2003a). Semantic processing in the left inferior prefrontal cortex: a combined functional magnetic resonance imaging and transcranial magnetic stimulation study. J. Cogn. Neurosci. 15, 71-84.

Devlin, J. T., Raley, J., Tunbridge, E., Lanary, K., Floyer-Lea, A., Narain, C., Cohen, I., Behrens, T., Jezzard, P., Matthews, P. M., and Moore, D. R. (2003b). Functional asymmetry for auditory processing in human primary auditory cortex. J. Neurosci. 23, 11516-11522.

Forman, S. D., Cohen, J.D., Fitzgerald, M., Eddy, W. F., Mintun, M. A., and Noll, D. C. (1995). Improved assessment of significant activation in functional magnetic resonance imaging (fMRI): use of a cluster-size threshold. Magn. Reson. Med. 33, 636-647.

Foster, N. E., and Zatorre, R. J. (2010). A role for the intraparietal sulcus in transforming musical pitch information. Cereb. Cortex 20, 1350-1359.

Frank, E., Landgrebe, M., Kleinjung, T., Hajak, G., and Langguth, B. (2010). Burst transcranial magnetic stimulation for the treatment of tinnitus. CNS Spectr. 15, 536-537.

Friston, K. J., Tononi, G., Reeke, G. N. Jr., Sporns, O., and Edelman, G. M. (1994). Value-dependent selection in the brain: simulation in a synthetic

\section{ACKNOWLEDGMENTS}

Jamila Andoh was supported by a fellowship from the MNI (CIBC), and an NSERC grant with R. Zatorre. The authors thank Nick Foster for advice regarding the design of the fMRI experiment and help with the melody task. The authors thank the volunteers for participating in the study, and the staff of the McConnell Brain Imaging Centre for their technical assistance.

neural model. Neuroscience 59, 229-243.

Gilio, F., Rizzo, V., Siebner, H. R., and Rothwell, J. C. (2003). Effects on the right motor hand-area excitability produced by low-frequency rTMS over human contralateral homologous cortex. J. Physiol. (Lond.) 551, 563-573.

Giraud, A. L., Kleinschmidt, A., Poeppel D., Lund, T. E., Frackowiak, R. S., and Laufs, H. (2007). Endogenous cortical rhythms determine cerebral specialization for speech perception and production. Neuron 56, 1127-1134.

Good, C. D., Johnsrude, I., Ashburner, J., Henson, R. N., Friston, K. J., and Frackowiak, R. S. (2001). Cerebral asymmetry and the effects of sex and handedness on brain structure: a voxel-based morphometric analysis of 465 normal adult human brains. Neuroimage 14, 685-700.

Gough, P. M., Nobre, A. C., and Devlin, J. T. (2005). Dissociating linguistic processes in the left inferior frontal cortex with transcranial magnetic stimulation. J. Neurosci. 25, 8010-8016.

Hall, D. A., Haggard, M. P., Akeroyd, M. A., Palmer, A. R., Summerfield, A. Q., Elliott, M. P., Gurney, E. M., and Bowtell, R.W. (1999). "Sparse” temporal sampling in auditory fMRI. Hum. Brain Mapp. 7, 213-223.

Hall, D.A., Summerfield, A. Q., Goncalves, M. S., Foster, J. R., Palmer, A. R., and Bowtell, R. W. (2000). Time-course of the auditory BOLD response to scanner noise. Magn. Reson. Med. 43, 601-606.

Hallett, M. (2000). Transcranial magnetic stimulation and the human brain. Nature 406, 147-150.

Herbsman, T., Forster, L., Molnar, C. Dougherty, R., Christie, D., Koola, J., Ramsey, D., Morgan, P. S., Bohning, D. E., George, M. S., and Nahas, Z. (2009). Motor threshold in transcranial magnetic stimulation: the impact of white matter fiber orientation and skull-to-cortex distance. Hum. Brain Mapp. 30, 2044-2055.

Hickok, G., and Poeppel, D. (2007). The cortical organization of speech processing. Nat. Rev. Neurosci. 8, 393-402.

Hoogendam, J. M., Ramakers, G. M. and Di Lazzaro, V. (2010). Physiology of repetitive transcranial magnetic stimulation of the human brain. Brain Stimul. 3, 95-118.
Hyde, K. L., Peretz, I., and Zatorre, R. J. (2008). Evidence for the role of the right auditory cortex in fine pitch resolution. Neuropsychologia 46, 632-639.

Jamison, H. L., Watkins, K. E., Bishop, D. V., and Matthews, P. M. (2006) Hemispheric specialization for processing auditory nonspeech stimuli. Cereb. Cortex 16, 1266-1275.

Jenkinson, M., and Smith, S. (2001). A global optimisation method for robust affine registration of brain images. Med. Image Anal. 5, 143-156.

Johnsrude, I. S., Penhune, V. B., and Zatorre, R. J. (2000). Functional specificity in the right human auditory cortex for perceiving pitch direction. Brain J. Neurol. 123(Pt 1), 155-163.

Knecht,S.,Ellger, T., Breitenstein, C., Bernd Ringelstein, E., and Henningsen, $\mathrm{H}$. (2003). Changing cortical excitability with low-frequency transcranial magnetic stimulation can induce sustained disruption of tactile perception. Biol. Psychiatry 53, 175-179.

Knecht, S., Floel, A., Drager, B., Breitenstein, C., Sommer, J., Henningsen, H., Ringelstein, E. B., and Pascual-Leone, A. (2002). Degree of language lateralization determines susceptibility to unilateral brain lesions. Nat. Neurosci. 5, 695-699.

Kulynych, J. J., Vladar, K., Jones, D. W., and Weinberger, D. R. (1994). Gender differences in the normal lateralization of the supratemporal cortex: MRI surface-rendering morphometry of Heschl's gyrus and the planum temporale. Cereb. Cortex 4, 107-118.

Loftus, G. R., and Masson, M. E. J. (1994). Using confidence intervals in within subject designs. Psychon. Bull. Rev. 1, 476-490.

Maccabee, P. J., Eberle, L., Amassian, V. E., Cracco, R. Q., Rudell, A., and Jayachandra, M. (1990). Spatial distribution of the electric field induced in volume by round and figure " 8 " magnetic coils: relevance to activation of sensory nerve fibers. Electroencephalogr. Clin. Neurophysiol. 76, 131-141.

Mathys, C., Loui, P., Zheng, X., and Schlaug, G. (2010). Non-invasive brain stimulation applied to Heschl's gyrus modulates pitch discrimination. Front. Psychol. 1:193. doi: 10.3389/ fpsyg.2010.00193 
Mills, K. R., Boniface, S. J., and Schubert, M. (1992). Magnetic brain stimulation with a double coil: the importance of coil orientation. Electroencephalogr. Clin. Neurophysiol. 85, 17-21.

Morosan, P., Rademacher, J., Schleicher,A., Amunts, K., Schormann, T., and Zilles, K. (2001). Human primary auditory cortex: cytoarchitectonic subdivisions and mapping into a spatial reference system. Neuroimage 13, 684-701.

O'shea, J., Johansen-Berg, H., Trief, D., Gobel, S., and Rushworth, M. F. S. (2007). Functionally specific in human premotor reorganization cortex. Neuron 54, 479-490.

Pascual-Leone, A., Tormos, J. M., Keenan, J., Tarazona, F., Canete, C., and Catala, M. D. (1998). Study and modulation of human cortical excitability with transcranial magnetic stimulation. J. Clin. Neurophysiol. 15, 333-343.

Patterson, R. D., Uppenkamp, S., Johnsrude, I. S., and Griffiths, T. D. (2002). The processing of temporal pitch and melody information in auditory cortex. Neuron 36, 767-776.

Peinemann, A., Reimer, B., Loer, C., Quartarone,A., Munchau,A., Conrad, B., and Siebner, H. R. (2004). Longlasting increase in corticospinal excitability after 1800 pulses of subthreshold $5 \mathrm{~Hz}$ repetitive TMS to the primary motor cortex. Clin. Neurophysiol. 115, 1519-1526.

Penhune, V. B., Zatorre, R. J., MacDonald, J. D., and Evans, A. C. (1996). Interhemispheric anatomical differences in human primary auditory cortex: probabilistic mapping and volume measurement from magnetic resonance scans. Cereb. Cortex 6, 661-672.

Peretz, I. (1990). Processing of local and global musical information by unilateral brain-damaged patients. Brain J. Neurol. 113(Pt 4), 1185-1205.

Poeppel, D. (2003). The analysis of speech in different temporal integration windows: cerebral lateralization as "asymmetric sampling in time." Speech Commun. 41, 245-255.

Puschmann, S., Uppenkamp, S., Kollmeier, B., and Thiel, C. M. (2010). Dichotic pitch activates pitch processing centre in Heschl's gyrus. Neuroimage 49, 1641-1649.

Rossi, S., Hallett, M., Rossini, P. M., and Pascual-Leone, A. (2009). Safety, ethical considerations, and application guidelines for the use of transcranial magnetic stimulation in clinical practice and research. Clin. Neurophysiol. 120, 2008-2039.

Rossi, S., Pasqualetti, P., Rossini, P. M., Feige, B., Ulivelli, M., Glocker, F. X., Battistini, N., Lucking, C. H., and Kristeva-Feige, R. (2000). Effects of repetitive transcranial magnetic stimulation on movement-related cortical activity in humans. Cereb. Cortex 10, 802-808.

Rothwell, J. C., Day, B. L., and Amassian, V.E. (1992). Near threshold electrical and magnetic transcranial stimuli activate overlapping sets of cortical neurones in humans. J. Physiol. 452, 109.

Rothwell, J. C., Hallett, M., Berardelli, A., Eisen, A., Rossini, P., and Paulus, W. (1999). Magnetic stimulation: motor evoked potentials. The International Federation of Clinical Neurophysiology. Electroencephalogr. Clin. Neurophysiol. Suppl. 52, 97-103.

Rounis, E., Lee, L., Siebner, H. R., Rowe, J. B., Friston, K. J., Rothwell, J. C., and Frackowiak, R. S. (2005). Frequency specific changes in regional cerebral blood flow and motor system connectivity following rTMS to the primary motor cortex. Neuroimage 26, 164-176.

Schambra, H. M., Sawaki, L., and Cohen, L. G. (2003). Modulation of excitability of human motor cortex (M1) by $1 \mathrm{~Hz}$ transcranial magnetic stimulation of the contralateral M1. Clin. Neurophysiol. 114, 130-133.

Schluter, N. D., Rushworth, M. F., Mills, K. R., and Passingham, R. E. (1999). Signal-, set-, and movement-related activity in the human premotor cortex. Neuropsychologia 37, 233-243.

Schmithorst, V. J., and Holland, S. K. (2007). Sex differences in the development of neuroanatomical functional connectivity underlying intelligence found using Bayesian connectivity analysis. Neuroimage 35, 406-419.

Schonwiesner, M., Rubsamen, R., and von Cramon, D. Y. (2005). Hemispheric asymmetry for spectral and temporal processing in the human antero-lateral auditory belt cortex. Eur. J. Neurosci. 22, 1521-1528.

Smith, S. M. (2002). Fast robust automated brain extraction. Hum. Brain Mapp. 17, 143-155.
Stewart, L., Ellison, A., Walsh, V., and Cowey, A. (2001). The role of transcranial magnetic stimulation (TMS) in studies of vision, attention and cognition. Acta Psychol. (Amst.) 107 275-291.

Stewart, L., von Kriegstein, K., Warren, J D., and Griffiths, T. D. (2006). Music and the brain: disorders of musical listening. Brain 129, 2533-2553.

Stokes, M. G., Chambers, C. D., Gould, I. C., Henderson, T. R., Janko, N. E. Allen, N. B., and Mattingley, J. B. (2005). Simple metric for scaling motor threshold based on scalp-cortex distance: application to studies using transcranial magnetic stimulation. $J$ Neurophysiol. 94, 4520-4527.

Strafella, A. P., and Paus, T. (2001). Cerebral blood-flow changes induced by paired-pulse transcranial magnetic stimulation of the primary motor cortex. J. Neurophysiol. 85, 2624-2629.

Strafella, A. P., Paus, T., Barrett, J., and Dagher, A. (2001). Repetitive transcranial magnetic stimulation of the human prefrontal cortex induces dopamine release in the caudate nucleus. J. Neurosci. 21, RC157.

Tallal, P., Miller, S., and Fitch, R. (1993) Neurobiological basis of speech: a case for the preeminence of temporal processing. Ann. N. Y.Acad. Sci.682,27-47.

Thiel, A., Habedank, B., Herholz, K. Kessler, J., Winhuisen, L., Haupt, W. F., and Heiss, W. D. (2006a). From the left to the right: how the brain compensates progressive loss of language function. Brain Lang. 98, 57-65.

Thiel, A., Schumacher, B., Wienhard, K. Gairing, S., Kracht, L. W., Wagner, R. Haupt, W. F., and Heiss, W. D. (2006b). Direct demonstration of transcallosal disinhibition in language networks. J. Cereb. Blood Flow Metab. 26, 1122-1127.

Wassermann, E. M., Grafman, J., Berry, C. Hollnagel, C., Wild, K., Clark, K., and Hallett, M. (1996). Use and safety of a new repetitive transcranial magnetic stimulator. Electroencephalogr. Clin. Neurophysiol. 101, 412-417.

Worsley, K. J., Chen, J. I., Lerch, J., and Evans, A. C. (2005). Comparing functional connectivity via thresholding correlations and singular value decomposition. Philos. Trans. R. Soc Lond. B Biol. Sci. 360, 913-920.
Worsley, K. J., Evans, A. C., Marrett, S., and Neelin, P. (1992). A three-dimensional statistical analysis for CBF activation studies in human brain. J. Cereb. Blood Flow Metab. 12, 900-918.

Zangen, A., Roth, Y., Voller, B., and Hallett, M. (2005). Transcranial magnetic stimulation of deep brain regions: evidence for efficacy of the H-coil. Clin. Neurophysiol. 116, 775-779.

Zatorre, R. J. (1988). Pitch perception of complex tones and human temporallobe function. J. Acoust. Soc. Am. 84, 566-572.

Zatorre, R. J., and Belin, P. (2001a). Spectral and temporal processing in human auditory cortex. Cereb. Cortex 11, 946-953.

Zatorre, R. J., and Belin, P. (2001b). Spectral and temporal processing in human auditory cortex. Cereb. Cortex 11, 946-953.

Zatorre, R. J., Belin, P., and Penhune, V. B. (2002). Structure and function of auditory cortex: music and speech. Trends Cogn. Sci. (Regul. Ed.)6,37-46.

Zatorre, R. J., and Gandour, J. T. (2008). Neural specializations for speech and pitch: moving beyond the dichotomies. Philos. Trans. R. Soc. Lond. B Biol. Sci. 363, 1087-1104.

Conflict of Interest Statement: The authors declare that the research was conducted in the absence of any commercial or financial relationships that could be construed as a potential conflict of interest.

Received: 14 January 2011; accepted: 27 June 2011; published online: 15 July 2011. Citation: Andoh J and Zatorre RJ (2011) Interhemispheric connectivity influences the degree of modulation of TMS induced effects during auditory processing. Front. Psychology 2:161. doi: 10.3389/ fpsyg.2011.00161

This article was submitted to Frontiers in Auditory Cognitive Neuroscience, a specialty of Frontiers in Psychology. Copyright (c) 2011 Andoh and Zatorre. This is an open-access article subject to a nonexclusive license between the authors and Frontiers Media SA, which permits use, distribution and reproduction in other forums, provided the original authors and source are credited and other Frontiers conditions are complied with. 